\title{
Absenteísmo-doença no serviço público municipal da Prefeitura Municipal de Vitória
} Sickness-absenteeism among municipal civil servants in Vitória, Brazil Vitor Guerzet Ayres Bastos', Patricia Grativol Costa Saraiva², Fábio Petersen Saraiva²

\begin{abstract}
RESUMO | Contexto: O conhecimento sobre as doenças que levam ao afastamento laboral constitui uma ferramenta importante para a elaboração de medidas que possam tornar o ambiente de trabalho mais seguro e agradável para o trabalhador, reduzindo o índice de absenteísmo-doença. Objetivo: Estudar as causas de absenteísmo-doença entre os servidores públicos da Prefeitura Municipal de Vitória (Espírito Santo, Brasil) no ano de 2012. Métodos: Estudo epidemiológico retrospectivo e descritivo. Os dados foram fornecidos pelo Serviço de Medicina Ocupacional da Prefeitura Municipal de Vitória. Resultados: As doenças dos aparelhos respiratório e osteomuscular e do tecido conjuntivo foram as principais causas de absenteísmo-doença entre os servidores municipais em 2012. No período avaliado, foram concedidas 14.271 licenças para tratamento de saúde com duração média de 10,2 dias. O percentual de absenteísmodoença encontrado foi de 4,79\%. Conclusão: Este trabalho demonstrou que as doenças dos aparelhos respiratório e osteomuscular e do tecido conjuntivo foram as principais causas de absenteísmo-doença no serviço público da Prefeitura Municipal de Vitória.
\end{abstract}

Palavras-chave I absenteísmo; setor público; licença médica; saúde do trabalhador.

ABSTRACT | Context: Knowing the diseases that lead to leave of absence is important for the development of measures capable of creating the conditions for a safer and more pleasant working environment for the workers, which may reduce sickness-absenteeism index. Objective: To identify the causes of sickness-absenteeism among municipal civil servants in Vitória (Espírito Santo, Brazil) in 2012. Methods: This is a descriptive and retrospective epidemiological study based on information disclosed by the Office of Occupational Medicine of the municipal government of Vitória. Results: Respiratory, musculoskeletal, and connective tissue disorders were the main causes of absenteeism among civil servants in 2012. During this period, 14,271 sick leaves were granted, with an average duration of 10.2 days. The percentage of absenteeism found was $4.79 \%$. Conclusion: The main causes of sickness-absenteeism among municipal civil servants in Vitória were diseases of the respiratory and musculoskeletal systems and connective tissue. Keywords I absenteeism; public sector; sick leave; occupational health. 


\section{INTRODUÇÃO}

O homem, nas empresas modernas, é considerado a principal peça do sistema de produção, visto que sua capacidade produtiva proporciona a competitividade da empresa e, consequentemente, o seu sucesso ${ }^{1}$. As condições sociais e de trabalho são determinantes na saúde das pessoas e na organização do trabalho, bem como estão associadas aos problemas de saúde do trabalhador. Dessa maneira, a submissão do trabalhador às demandas do sistema produtivo gera desdobramentos que extrapolam o ambiente e as relações de trabalho. Essa condição impõe condicionantes ao estilo e à forma de viver do ser que trabalha, com impactos negativos no plano familiar e social, em razão de a maior parte do seu tempo cotidiano ser dedicada ao trabalho em detrimento da sua vida privada, do lazer e dos cuidados com a própria saúde. O corpo do trabalhador é afetado com a inserção na vida produtiva, cujas atividades caracterizam-se por um exercício rotineiro de gestos, posturas e atividades mentais que o obrigam ao abandono ou ao descaso com o corpo ${ }^{2,3}$.

Por meio do conhecimento sobre as doenças mais prevalentes nas licenças médicas dos trabalhadores, será possível colaborar para tornar o ambiente de trabalho menos agressivo, mais humano e seguro, aumentando o grau de satisfação do trabalhador com suas atividades profissionais e diminuindo o índice de absenteísmo. Esse conhecimento permite uma visão mais detalhada, tanto individual quanto epidemiológica, das condições de trabalho, favorecendo estudos científicos que subsidiem a gerência de Recursos Humanos e o serviço de Medicina do Trabalho, a fim de reavaliar a política institucional com o objetivo de melhorar a saúde do trabalhador ${ }^{4,5}$.

O objetivo deste estudo foi identificar as causas de absenteísmo-doença mais prevalentes que acometem os trabalhadores do serviço público da Prefeitura Municipal de Vitória, assim como a duração das licenças por doenças especificadas no diagnóstico médico e o perfil do servidor absenteísta. Os dados encontrados poderão constituir subsídios para o aprimoramento das questões que dizem respeito à saúde do trabalhador, criando possibilidades de prevenção de doenças, redução do absenteísmo, melhorias das condições de trabalho e tratamento de doenças.

\section{MÉTODOS}

Trata-se de um estudo epidemiológico retrospectivo e descritivo sobre a ocorrência de afastamentos de servidores públicos civis da Prefeitura Municipal de Vitória por diversos transtornos.

A instituição campo de estudo foi a Coordenação de Medicina do Trabalho (CMT). A CMT está subordinada à Gerência de Saúde e Apoio Social ao Servidor (GSAS), da Secretaria Municipal de Administração (SEMAD).

A população desta pesquisa foi constituída por todos os servidores dos diversos setores da administração pública da Prefeitura Municipal de Vitória que, no período de janeiro a dezembro de 2012, tiveram registro de afastamento do trabalho conforme códigos presentes na Classificação Internacional de Doenças (CID-10) ${ }^{6}$.

Esta pesquisa foi realizada por meio de um banco de dados fornecido pela GSAS, elaborado com base nos dados contidos na ficha de atendimento do servidor do sistema de arquivo virtual da CMT. O banco de dados em questão foi gentilmente fornecido em formato de planilha eletrônica após a autorização da Subsecretaria Municipal de Gestão de Pessoas. O instrumento foi elaborado visando atender aos objetivos da pesquisa, sendo os dados divididos em três itens: caracterização do trabalhador (sexo, data de nascimento, idade); prática profissional (categoria profissional, setor de lotação, data de admissão e característica do contrato de trabalho); e saúde ocupacional (CID, necessidade de afastamento, número de dias concedidos). É importante salientar que no banco de dados fornecido não constavam quaisquer informações pessoais (nome ou matrícula) dos servidores da prefeitura, preservando, dessa maneira, o sigilo médico.

Foram ainda solicitadas informações sobre a composição do quadro funcional, por tipo de vínculo, por meio do canal eletrônico Sistema de Informações ao Cidadão (SIC), disponibilizado pela prefeitura de Vitória em seu portal na internet. No período que abrangeu a pesquisa, o quadro funcional da prefeitura de Vitória era composto por 12.299 funcionários, sendo 10.196 estatutários (efetivos), 512 celetistas, 468 comissionados e 1.123 contratados por tempo determinado. $\mathrm{O}$ processo de obtenção dos dados para o estudo compreendeu novembro de 2013 a janeiro de 2014.

Para obtenção de embasamento teórico e dados comparativos, foram consultadas produções científicas em bibliotecas e bancos de dados virtuais, como Literatura Latino-Americana e 
do Caribe em Ciências da Saúde (LILACS), Scientific Electronic Library Online (SciELO) e Medical Literature Analysis and Retrieval System Online (MEDLINE), além de sítios eletrônicos de revistas de produção científica e instituições, como Organização Internacional do Trabalho(OIT), Ministério da Saúde do Brasil, Associação Nacional de Medicina do Trabalho (ANAMT) e Organização Mundial de Saúde (OMS).

As recomendações do Subcomitê de Absenteísmo da Sociedade Internacional de Saúde Ocupacional ${ }^{7}$ foram utilizadas neste estudo para se obter os indicadores e comparar os dados do absenteísmo. Os índices de frequência, gravidade, percentual de absenteísmo e duração média das ausências foram obtidos obedecendo às seguintes fórmulas:

- Índice de frequência = No licenças médicas $/$ Efetivo

- Índice de gravidade $=$ Dias Perdidos $/$ Efetivo

- Percentual de absenteísmo = Dias Perdidos x 100/ No servidores $\mathrm{x}$ No dias úteis

- Duração média das ausências $=$ No dias de afastamento $/$ No licenças médicas

Para determinação do número de dias úteis utilizado no cálculo do percentual de absenteísmo, foram desconsiderados feriados, pontos facultativos decretados pela prefeitura, sábados e domingos. Dessa forma, para o cálculo foi considerado o número de 246 dias úteis em 2012.

Os dados e o conteúdo deste estudo foram organizados utilizando os softwares Exce $^{\circledR}{ }^{\text {e Word }}{ }^{\circledR}$ do pacote Microsoft Office ${ }^{\circledR}$ for Mac 2011.

\section{RESULTADOS}

No período de 01 de janeiro de 2012 a 31 de dezembro de 2012, foram realizados 18.426 atendimentos médico-ocupacionais na Coordenação de Medicina do Trabalho da Prefeitura Municipal de Vitória. Desse total, 0,52\% das licenças médicas foi negado pelo serviço médico pericial.

Os dados oferecidos pelo setor para este estudo não especificam o motivo da negativa da licença médica, apenas o CID relacionado com o evento e a conclusão do médico perito, que no banco de dados está limitado ao conteúdo de "licença negada". Os grupos de doenças, organizados pelo CID- $10^{6}$, que mais frequentemente foram motivo de negativa de licença médica são: com $43 \%$, os fatores que influenciam o estado de saúde e o contato com os serviços de saúde (CID Z00-Z99); e, com 15\%, os transtornos mentais e comportamentais. O Gráfico 1 representa esses grupos de doenças envolvidos na negativa de licença médica.

As licenças para tratamento de saúde, licença maternidade e licença para acompanhamento de familiar necessitam de homologação no serviço médico. Para determinação do absenteísmo-doença, este estudo considerou somente as licenças para tratamento de saúde do próprio servidor. Dessa maneira, a licença maternidade e a licença para acompanhamento de familiar, que constavam no relatório original, foram desconsideradas para traçar o perfil de absenteísmo-doença.

Do total de 18.426 atendimentos realizados no ano de 2012, foram concedidas 14.271 licenças para tratamento de saúde (absenteísmo-doença). A menor licença concedida foi de 1 dia, e a maior, de 366 dias de afastamento. A duração média dos afastamentos foi de 10,2 dias. Quando somados, os afastamentos atingem a marca de 144.940 dias de trabalho perdidos. Osíndices desses afastamentos estão apresentados no Quadro 1.

A Tabela 1 traça o perfil do servidor municipal absenteísta por doença, com relação aos números absolutos e relativos

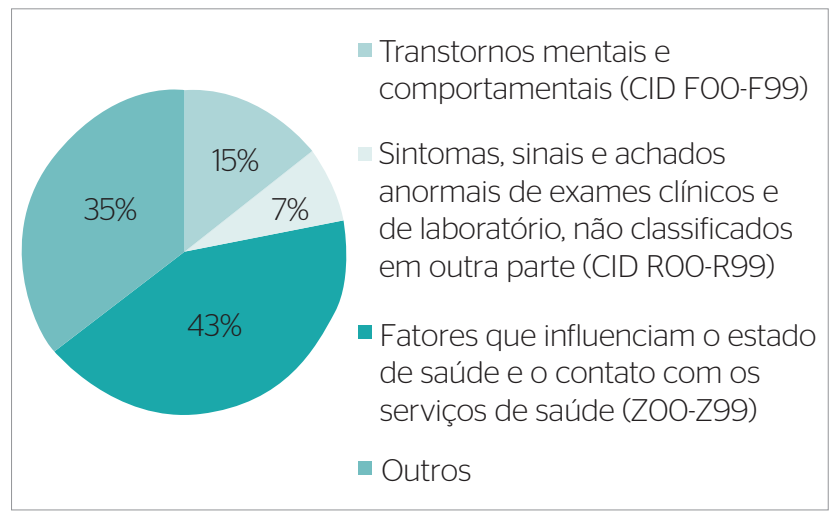

Gráfico 1. Grupos de doenças relacionadas à negativa de licença médica dos servidores municipais de Vitória, em 2012.

Quadro 1. Absenteísmo-doença do servidor municipal de Vitória, em 2012.

\begin{tabular}{|cc|}
\hline \multicolumn{2}{|c|}{ Absenteísmo-doença } \\
\hline Número de licenças & 14.271 \\
\hline Total de dias perdidos & 144.940 \\
\hline Índice de frequência & 1,2 \\
\hline Índice de gravidade & 11,8 \\
\hline Absenteísmo-doença & $4,79 \%$ \\
\hline Duração média (dias) & 10,2 \\
\hline
\end{tabular}


de licenças médicas e à duração média desses afastamentos no período estudado.

A Tabela 2 apresenta os indicadores de absenteísmo-doença dos servidores quanto ao vínculo de trabalho.

Para compreender a distribuição da ocorrência das licenças médicas por doença no ano de 2012, pode-se observar o Gráfico 2. Constata-se que houve um aumento da ocorrência nos meses de junho a agosto, com pico de 1.578 licenças no mês de agosto. Em janeiro, foi registrado o menor valor, 577 licenças. A média de licenças concedidas por mês correspondeu a 1189,2.

O Gráfico 3 demonstra a duração média das licenças médicas por doenças dos servidores municipais ao longo do ano de 2012. Nota-se que o pico ocorreu no mês de janeiro (17,1 dias).

A distribuição da ocorrência de licenças em relação ao dia da semana em que ocorreu o atendimento médico na CMT pode ser visualizada no Gráfico 4 .

Nota-se que não existiram grandes variações no número de licenças concedidas ao longo da semana, exceto pela pequena elevação notada na quarta-feira.

Todas as licenças concedidas pela CMT são registradas com um CID associado. Dessa maneira, pode-se determinar qual a doença causadora do afastamento do servidor. Assim, as causas de absenteísmo-doença dos servidores municipais de Vitória no ano de 2012 foram organizadas quanto ao grupo/capítulo do CID-10 (Gráfico 5 e Tabela 3).

Observa-se que as doenças do aparelho respiratório (CID J00-J99) e as doenças do sistema osteomuscular e do tecido conjuntivo (CID M00-M99) foram as principais causas de afastamento por doença dos servidores municipais em 2012. As doenças do aparelho respiratório (CID J00-J99) apresentaram duração média de afastamento de 6,2 dias, enquanto as doenças do sistema osteomuscular e do tecido conjuntivo causaram um afastamento médio de 12,8 dias.

Tabela 1. Perfil do servidor municipal absenteísta por doença, quanto ao gênero, à idade e ao tempo de serviço, no ano de 2012.

\begin{tabular}{|c|c|c|c|}
\hline & Duração & \multicolumn{2}{|c|}{ Licenças médicas } \\
\cline { 3 - 4 } média (dias) & $n$ & $\%$
\end{tabular}

Gênero

\begin{tabular}{lccc}
\hline Feminino & 9,9 & 11.562 & 81,02 \\
\hline Masculino & 11,1 & 2.709 & 18,98 \\
\hline Geral & 10,2 & 14.271 & 100,00 \\
\hline
\end{tabular}

Faixa etária (anos)

\begin{tabular}{cccc}
\hline 20 a 29 & 4,9 & 1.245 & 8,72 \\
\hline 30 a 39 & 7,7 & 5.053 & 35,41 \\
\hline 40 a 49 & 10,5 & 4.447 & 31,16 \\
\hline 50 a 59 & 14,3 & 2.761 & 19,35 \\
\hline 60 a 69 & 17,5 & 744 & 5,21 \\
\hline 70 a 79 & 39,9 & 16 & 0,11 \\
\hline 80 a 89 & 4,8 & 5 & 0,04 \\
\hline Geral & 10,2 & 14.271 & 100,00 \\
\hline
\end{tabular}

Tempo de serviço (anos)

\begin{tabular}{cccc}
\hline O a 5 & 7,2 & 7.724 & 54,12 \\
\hline 5 a 10 & 11,4 & 3.436 & 24,08 \\
\hline 10 a 15 & 13,5 & 784 & 5,49 \\
\hline 15 a 20 & 17,1 & 697 & 4,88 \\
\hline 20 a 25 & 12,9 & 825 & 5,78 \\
\hline 25 a 30 & 20,5 & 529 & 3,71 \\
\hline 30 a 35 & 23,7 & 259 & 1,81 \\
\hline 35 a 40 & 14,1 & 15 & 0,11 \\
\hline 40 a 45 & 3,5 & 2 & 0,01 \\
\hline Geral & 10,2 & 14.271 & 100,00 \\
\hline
\end{tabular}

Tabela 2. Servidor municipal absenteísta por doença, quanto ao vínculo trabalhista, no ano de 2012.

\begin{tabular}{|c|c|c|c|c|c|c|}
\hline \multirow{2}{*}{ Forma de contrato de trabalho } & \multicolumn{2}{|c|}{ Licenças médicas } & \multirow{2}{*}{ IF } & \multirow{2}{*}{ IG } & \multirow{2}{*}{$\begin{array}{l}A D \\
(\%)\end{array}$} & \multirow{2}{*}{$\begin{array}{c}\text { DM } \\
\text { (dias) }\end{array}$} \\
\hline & $\mathrm{n}$ & $\%$ & & & & \\
\hline Estatutário (efetivo) & 11.761 & 82,41 & 1,15 & 13,18 & 5,36 & 11,4 \\
\hline Contrato por tempo determinado & 1.934 & 13,55 & 1,72 & 6,91 & 2,81 & 4,0 \\
\hline Celetista & 353 & 2,47 & 0,69 & 3,45 & 1,40 & 5,0 \\
\hline Comissionado & 137 & 0,96 & 0,29 & 2,27 & 0,92 & 7,7 \\
\hline Geral & 14271 & 100,00 & 1,16 & 11,78 & 4,79 & 10,2 \\
\hline
\end{tabular}

IF: índice de frequência; IG: índice de gravidade; AD: absenteísmo-doença; DM: duração média. 
A Tabela 4 apresenta os diagnósticos dos grupos de doença (CID-10) que, com maior frequência, causaram afastamentos para tratamento de saúde. Os valores relativos (\%) estão calculados em relação ao número geral de licenças por doença (14.271).
A distribuição da ocorrência e a duração média das licenças por doença dos servidores podem ser analisadas quanto à secretaria ou ao órgão municipal ao qual o cargo está vinculado na prefeitura municipal de Vitória (Tabela 5).

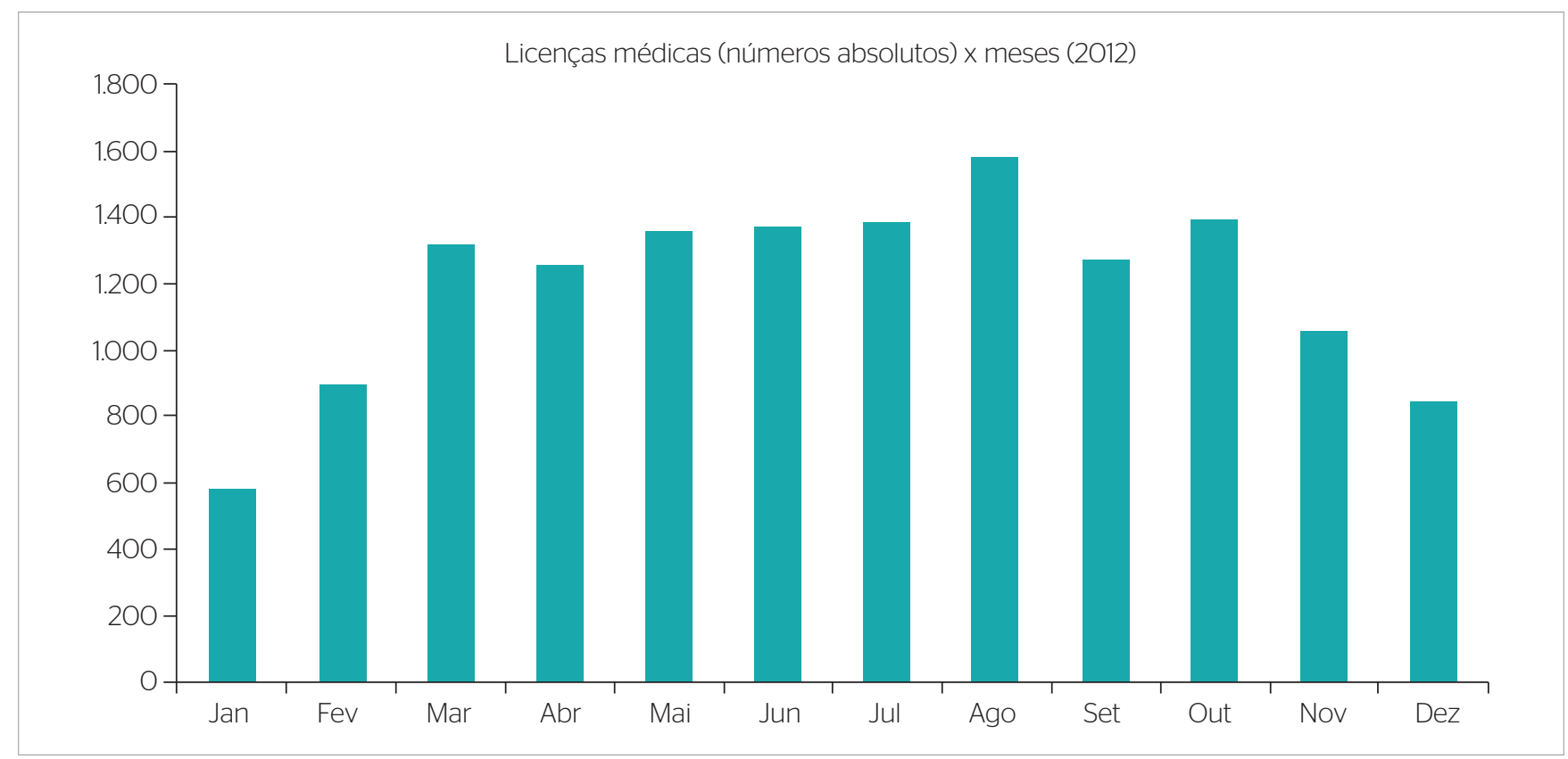

Gráfico 2. Distribuição da ocorrência de licenças médicas por doença em servidores municipais nos meses de 2012.

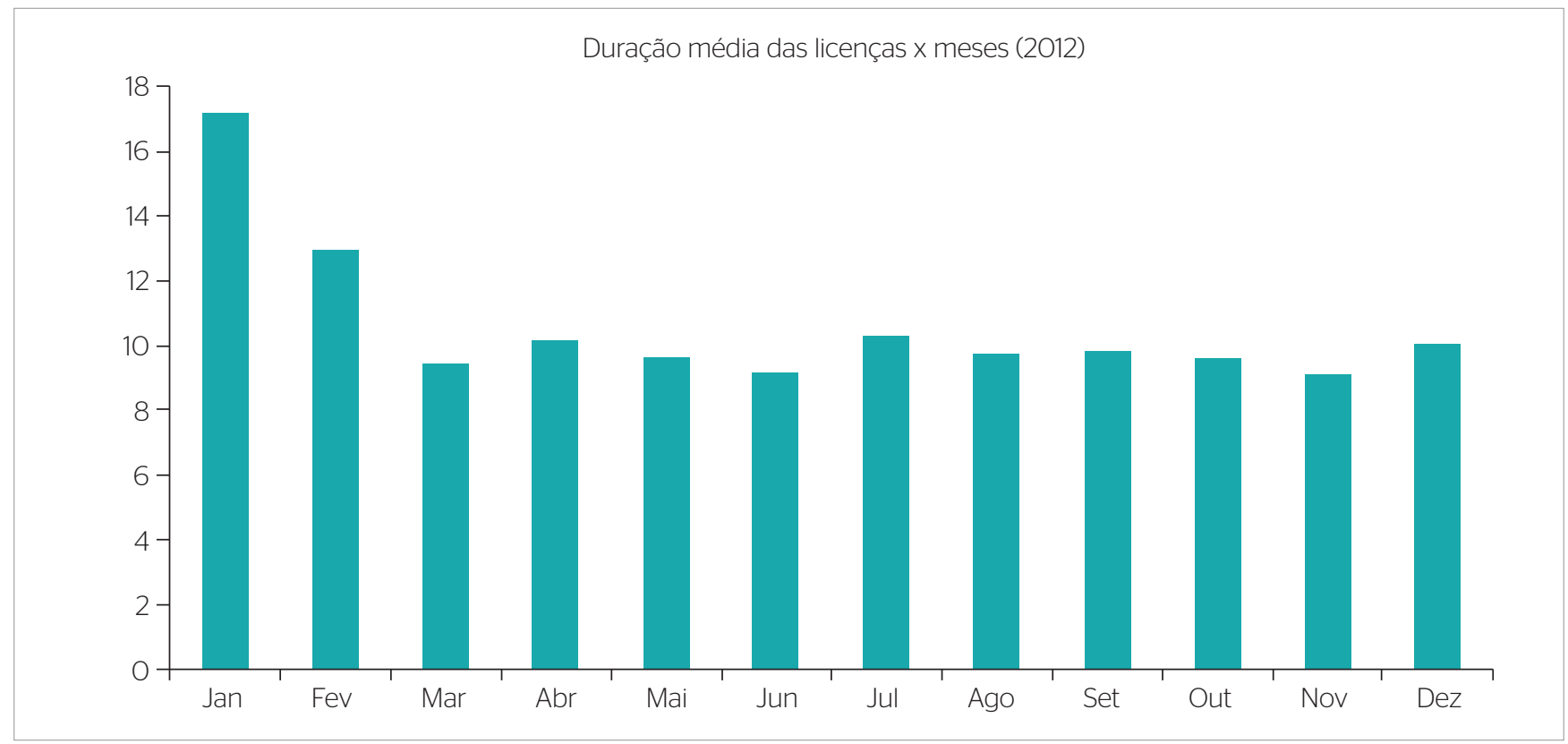

Gráfico 3. Distribuição da duração média das licenças médicas por doença em servidores municipais em relação aos meses de 2012. 
Licenças médicas (números absolutos) x dias da semana

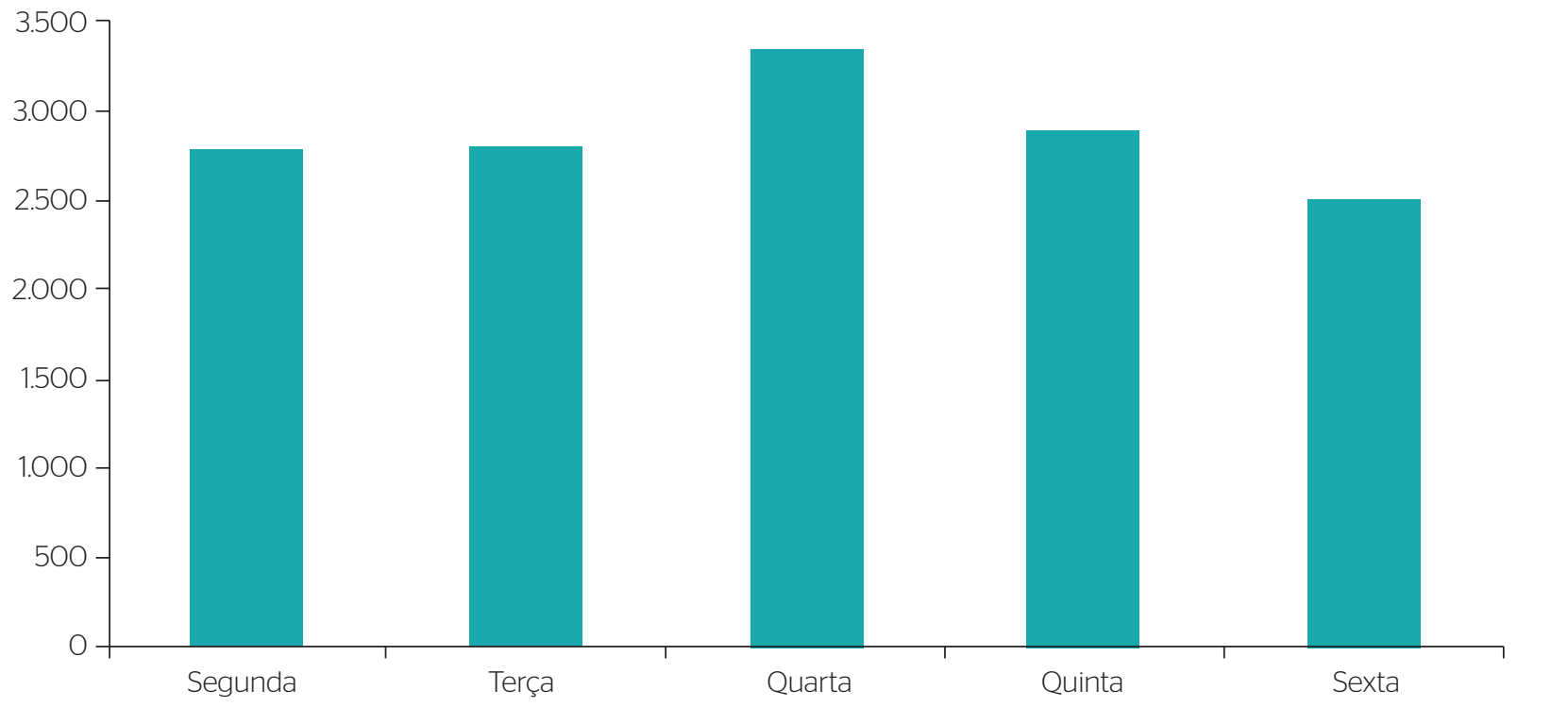

Gráfico 4. Distribuição da ocorrência das licenças médicas por doença, em relação aos dias da semana, dos servidores municipais, em 2012.

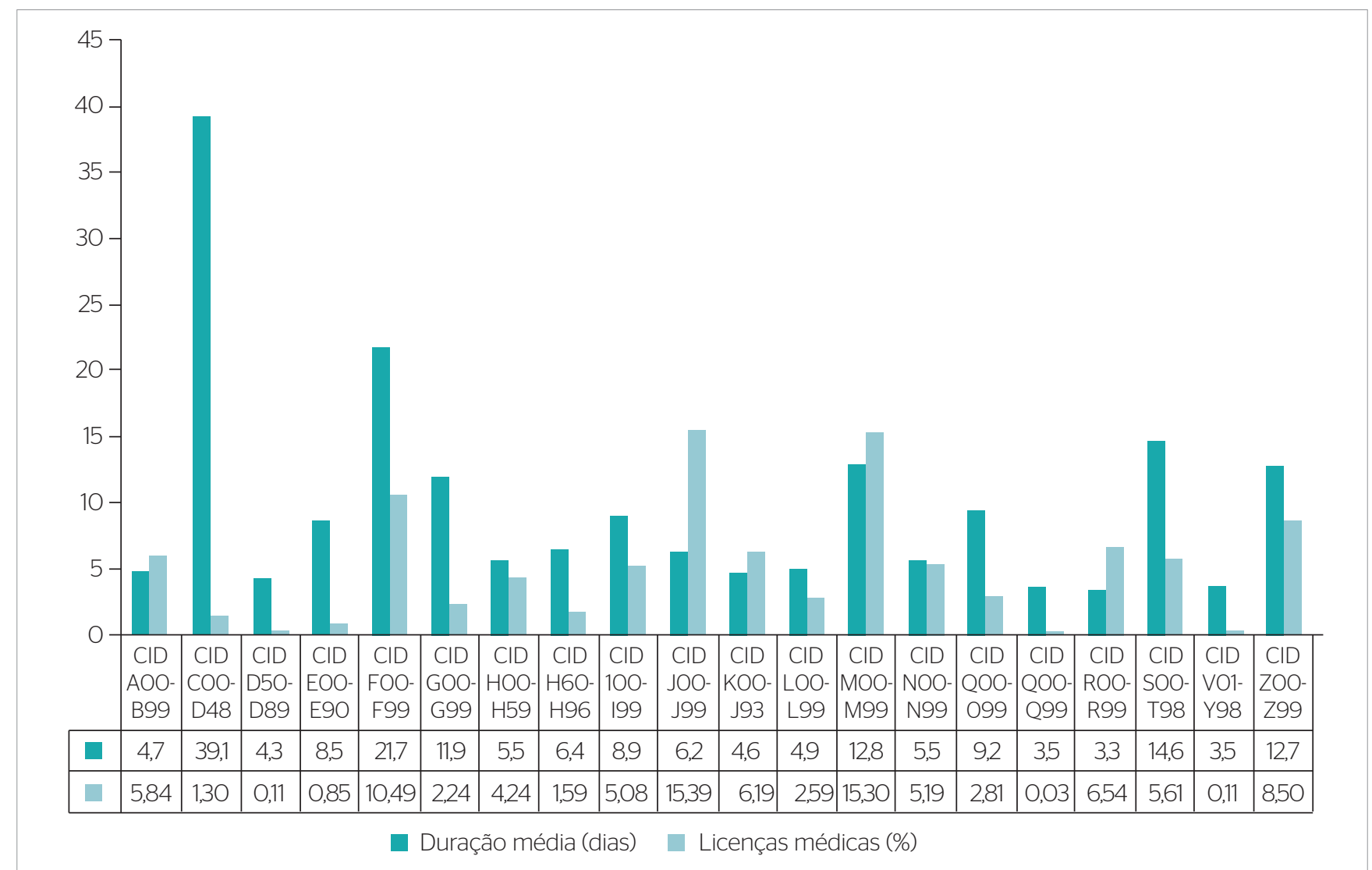

Gráfico 5. Causas de afastamento por doenças, organizadas segundo os capítulos da Classificação Internacional de Doenças, dos servidores municipais, em 2012. 
Tabela 3. Causas de afastamento por doenças, organizadas quanto aos capítulos da Classificação Internacional de Doenças, dos servidores municipais, em 2012.

\begin{tabular}{|c|c|c|c|}
\hline \multirow{2}{*}{ Grupo de doenças (CID-10) } & \multirow{2}{*}{$\begin{array}{l}\text { Duração } \\
\text { média (dias) }\end{array}$} & \multicolumn{2}{|c|}{ Licenças médicas } \\
\hline & & $\mathrm{n}$ & $\%$ \\
\hline Algumas doenças infecciosas e parasitárias (CID AO0-B99) & 4,7 & 834 & 5,84 \\
\hline Neoplasias [tumores] (CID COO-D48) & 39,1 & 186 & 1,30 \\
\hline Doenças do sangue e dos órgãos hematopoéticos e alguns transtornos imunitários (CID D50-D89) & 4,3 & 16 & 0,11 \\
\hline Doenças endócrinas, nutricionais e metabólicas (CID EOO-E9O) & 8,5 & 122 & 0,85 \\
\hline Transtornos mentais e comportamentais (CID FOO-F99) & 21,7 & 1497 & 10,49 \\
\hline Doenças do sistema nervoso (CID G00-G99) & 11,9 & 320 & 2,24 \\
\hline Doenças do olho e anexos (CID HOO-H59) & 5,5 & 605 & 4,24 \\
\hline Doenças do ouvido e da apófise mastoide (CID H6O-H96) & 6,4 & 227 & 1,59 \\
\hline Doenças do aparelho circulatório (CID IOO-199) & 8,9 & 725 & 5,08 \\
\hline Doenças do aparelho respiratório (CID JOO-J99) & 6,2 & 2197 & 15,39 \\
\hline Doenças do aparelho digestivo (CID KOO-K93) & 4,6 & 884 & 6,19 \\
\hline Doenças da pele e do tecido celular subcutâneo (CID LOO-L99) & 4,9 & 369 & 2,59 \\
\hline Doenças do sistema osteomuscular e do tecido conjuntivo (CID MOO-M99) & 12,8 & 2183 & 15,30 \\
\hline Doenças do aparelho geniturinário (CID NOO-N99) & 5,5 & 740 & 5,19 \\
\hline Gravidez, parto e puerpério (CID O00-099) & 9,2 & 401 & 2,81 \\
\hline Malformações congênitas, deformidades e anomalias cromossômicas (CID QOO-Q99) & 3,5 & 4 & 0,03 \\
\hline $\begin{array}{l}\text { Sintomas, sinais e achados anormais de exames clínicos e de laboratório, } \\
\text { não classificados em outra parte (CID ROO-R99) }\end{array}$ & 3,3 & 933 & 6,54 \\
\hline Lesões, envenenamentos e algumas outras consequências de causas externas (CID SOO-T98) & 14,6 & 800 & 5,61 \\
\hline Causas externas de morbidade e de mortalidade (CID V01-Y98) & 3,5 & 15 & 0,11 \\
\hline Fatores que influenciam o estado de saúde e o contato com os serviços de saúde (CID ZOO-Z99) & 12,7 & 1213 & 8,50 \\
\hline Geral & 10,2 & 14271 & 100,00 \\
\hline
\end{tabular}

CID-10: Classificação Internacional de Doenças.

Tabela 4. Doenças como causas de afastamento dos servidores municipais, em 2012.

\begin{tabular}{|c|c|c|c|}
\hline \multirow{2}{*}{ Diagnóstico } & \multirow{2}{*}{$\begin{array}{c}\text { Duração } \\
\text { média (dias) }\end{array}$} & \multicolumn{2}{|c|}{ Licenças médicas } \\
\hline & & $\mathrm{n}$ & $\%$ \\
\hline \multicolumn{4}{|c|}{ Doenças do aparelho respiratório (CID JOO-J99) } \\
\hline Sinusites & 2,9 & 681 & 4,77 \\
\hline Amigdalites & 2,6 & 219 & 1,53 \\
\hline Nasofaringites agudas & 2,1 & 186 & 1,30 \\
\hline Faringites & 2,5 & 177 & 1,24 \\
\hline Doenças das cordas vocais & 30,5 & 140 & 0,9 \\
\hline Influenza & 2,4 & 137 & 0,96 \\
\hline Laringites crônicas & 21,4 & 128 & 0,90 \\
\hline Laringites agudas & 3,4 & 99 & 0,69 \\
\hline Asma & 4,4 & 69 & 0,48 \\
\hline Outras & 6,1 & 361 & 2,53 \\
\hline Geral (CID JOO-J99) & 6,2 & 2197 & 15,39 \\
\hline
\end{tabular}


Tabela 4. Continuação.

Diagnóstico
Duração média (dias)
Licenças médicas

n

Doenças do sistema osteomuscular e do tecido conjuntivo (CID MOO-M99)

\begin{tabular}{|c|c|c|c|}
\hline Dorsalgias & 8,0 & 911 & 6,38 \\
\hline Sinovites e tenossinovites & 11,4 & 269 & 1,88 \\
\hline Outros transtornos articulares & 10,2 & 184 & 1,29 \\
\hline Lesões de ombro & 26,2 & 147 & 1,03 \\
\hline Transtornos internos dos joelhos & 17,1 & 133 & 0,93 \\
\hline Transtornos dos discos intervertebrais & 18,0 & 89 & 0,62 \\
\hline Mialgias & 14,1 & 75 & 0,53 \\
\hline Outros & 18,4 & 375 & 2,63 \\
\hline Geral (CID MOO-M99) & 12,8 & 2183 & 15,30 \\
\hline \multicolumn{4}{|c|}{ Transtornos mentais e comportamentais (CID FOO-F99) } \\
\hline Transtorno depressivo & 24,6 & 574 & 4,02 \\
\hline Reações ao estresse & 12,4 & 211 & 1,48 \\
\hline Outros transtornos ansiosos & 12,1 & 192 & 1,35 \\
\hline Transtorno afetivo bipolar & 40,3 & 139 & 0,97 \\
\hline Transtorno misto ansioso e depressivo & 23,6 & 95 & 0,67 \\
\hline Transtornos fóbico-ansiosos & 18,0 & 59 & 0,41 \\
\hline Ansiedade generalizada & 10,8 & 59 & 0,41 \\
\hline Transtorno de pânico & 18,5 & 28 & 0,20 \\
\hline Outros & 23,8 & 140 & 0,98 \\
\hline Geral (CID FOO-F99) & 21,7 & 1.497 & 10,49 \\
\hline Geral & 10,2 & 14.271 & 100,00 \\
\hline
\end{tabular}

CID-10: Classificação Internacional de Doenças.

Tabela 5. Distribuição da ocorrência de licenças médicas por doença, organizada por órgãos/secretarias, da Prefeitura Municipal de Vitória, em 2012.

\begin{tabular}{|c|c|c|c|}
\hline \multirow{2}{*}{ Órgão/secretaria } & \multirow{2}{*}{$\begin{array}{c}\text { Duração } \\
\text { média (dias) }\end{array}$} & \multicolumn{2}{|c|}{ Licenças médicas } \\
\hline & & $\mathrm{n}$ & $\%$ \\
\hline Secretaria de Educação & 10,0 & 8.384 & 58,75 \\
\hline Secretaria Municipal de Saúde & 9,3 & 4.127 & 28,92 \\
\hline Secretaria Municipal de Segurança Urbana & 7,7 & 824 & 5,77 \\
\hline Secretaria Municipal de Administração & 29,2 & 194 & 1,36 \\
\hline Secretaria Municipal de Fazenda & 15,5 & 125 & 0,88 \\
\hline Secretaria Municipal de Meio Ambiente & 9,0 & 101 & 0,71 \\
\hline Outras secretarias ou órgãos & 15,9 & 516 & 3,62 \\
\hline Geral & 10,2 & 14.271 & 100,00 \\
\hline
\end{tabular}




\section{DISCUSSÃO}

Não foram encontrados na literatura dados que pudessem servir de parâmetro no que diz respeito à negativa de licenças médicas. Dessa maneira, este estudo não pretende discutir a representatividade do resultado obtido, mas servir como referencial para futuros estudos.

Os indicadores de absenteísmo por doença, encontrados neste estudo, foram, em geral, maiores que os verificados nos servidores de Goiânia, no período de 2005 a 2010: índice de frequência de 0,32 ; índice de gravidade de 7,63; duração média das licenças de 23 dias; percentual de absenteísmo de $3 \%$. No ano de 2009, os servidores municipais de Curitiba apresentaram percentual de absenteísmo-doença de 5,19\% e duração média das licenças de 7,2 dias ${ }^{8,9}$.

Dessa maneira, os servidores municipais capixabas apresentaram menor duração média das licenças (10,2 dias) em relação aos goianos e maior em relação aos curitibanos. Já o percentual de absenteísmo-doença encontrado foi superior ao de Goiânia e inferior ao de Curitiba. Em 2012, os índices de frequência $(1,2)$ e de gravidade $(11,8)$ foram maiores em Vitória do que em Goiânia ${ }^{8}$.

Estudos realizados em países do Reino Unido apontam percentuais de absenteísmo-doença em servidores públicos que variam de 3,5\% (Escócia, em 2003 e 2004) a 6,5\% (Irlanda do Norte, em 2004 e 2005). O percentual de absenteísmo-doença aceito pela OIT é de 2,5\% $\%^{8,10}$.

O percentual de absenteísmo-doença encontrado no estudo é considerado alto, quando comparado com o percentual aceito pela OIT; porém, quando comparado aos números encontrados na literatura, possui a mesma magnitude. É possível, portanto, que o índice aceito pela OIT esteja subestimado e fora da realidade.

A predominância do gênero feminino $(81,02 \%)$ quanto à ocorrência de absenteísmo-doença não representou surpresa. $\mathrm{Na}$ literatura, o afastamento do trabalho pelo gênero feminino é apontado, na maioria dos estudos, como predominante. Os valores relativos apontados nesses estudos variam de 56,97 a $89,90 \% \%^{8,9,11-14}$. No tocante à duração média das licenças médicas, os servidores do sexo masculino receberam licenças com duração média maior (11,1 dias), em relação ao sexo feminino (9,9 dias).

Em relação à faixa etária, os dados encontrados coincidem com outros estudos em populações semelhantes ${ }^{8,9,11}$. No tocante ao tempo de vínculo com a instituição, os dados obtidos estão semelhante a outro estudo prévio na cidade de Vitória, que demonstrou maior parcela de afastamento entre os servidores com até cinco anos de vínculo institucional ${ }^{11}$.

Poucos estudos correlacionaram o tipo de vínculo de trabalho com os indicadores de absenteísmo ${ }^{11,12,15}$. Os resultados de nosso trabalho, assim como esses poucos estudos publicados, demonstraram que o trabalhador estatuário tende a se afastar com mais frequência do trabalho do que os demais. Essa diferença entre as categorias pode ser explicada pela tranquilidade que o servidor estatutário tem ao se afastar do trabalho, uma vez que goza de estabilidade. Tal pensamento é compartilhado por Reis et al., considerando afirmação prévia de que as taxas de incapacidade laboral reduzem-se pelo medo de dispensa do trabalho ${ }^{15}$.

Assim como nosso trabalho, Santos e Mattos também observaram uma maior ocorrência de licenças médicas por doença nos servidores nos meses de inverno, sobretudo em agosto $^{7}$. Já o estudo de Guimarães ${ }^{4}$, que avaliou os servidores de um hospital militar, apontou uma maior ocorrência nos meses de abril e maio.

Em relação à distribuição da duração média das licenças ao longo do ano, a literatura não dispunha de dados que pudessem ser comparados. Dessa maneira, este estudo não pôde discutir a relevância desse resultado, exceto para servir de referência para futuros estudos, considerando o pioneirismo de tal informação. Quanto à distribuição da ocorrência de licenças médicas pelo dia da semana, nossos achados divergem dos encontrados pelo estudo de Carneiro ${ }^{16}$, que apontou uma concentração das ocorrências nas segundas e sextas-feiras.

Assim como já identificado em outro estudo realizado no município de Vitória, também observamos que as doenças dos aparelhos respiratório e osteomuscular e do tecido conjuntivo foram as principais causas de absenteísmo-doença nos servidores municipais dessa cidade ${ }^{11}$. Esses achados contrastam com estudos realizados em outras capitais brasileiras, que registraram os transtornos mentais e comportamentais como as principais causas de afastamento por doença em suas populações ${ }^{7-9}$. É provável que a maior representatividade de afastamentos por doenças respiratórias no município de Vitória esteja relacionada aos altos índices de poluição atmosférica associados ao clima local ${ }^{17-19}$.

Os resultados obtidos neste estudo apontaram que as Secretarias de Educação e Saúde foram responsáveis 
pela maioria absoluta dos afastamentos por doenças entre os servidores municipais de Vitória no ano de 2012. Esses números se assemelham a estudos realizados com servidores municipais de Goiânia, Curitiba e Porto Alegre ${ }^{7-9,12}$.
Este trabalho detalhou as principais causas de absenteísmo-doença do serviço público da Prefeitura Municipal de Vitória. Esse conhecimento é fundamental para a organização e o planejamento de ações de promoção da saúde do trabalhador.

\section{REFERÊNCIAS}

1. Coelho MP, Oliveira MA, Araújo VE, Carvalho CM. Absenteísmo por causas odontológicas em uma empresa agropecuária da Região Sudeste do Estado de Minas Gerais. Rev Bras Pesq Saúde. 2010;12(1):14-8.

2. Sousa-Uva A, Serranheira F. Saúde e trabalho/(Doença): o desafio sistemático da prevenção dos riscos profissionais e o esquecimento reiterado da promoção da saúde. Rev Bras Med Trab. 2013;11(1):43-9.

3. Mergener CR, Kehrig RT, Traebert J.Sintomatologia músculo-esquelética relacionada ao trabalho esua relação com qualidadede vidaem bancários do meio oeste catarinense. Saúde Soc. São Paulo 2008;17(4):171-81.

4. Guimarães RSO, Castro HA. O absenteísmo entre os servidores civis de um hospital militar. Pesq Nav. 2007;20:74-78.

5. Balasteghin FSMM, Marrone LC, Silva-Junior JS. Absenteísmodoença de curta duração entre operadores de telemarketing. Rev Bras Med Trab. 2014;12(1):16-20.

6. Organização Mundial de Saúde (OMS). Classificação internacional de doenças e problemas relacionados à saúde: 10a revisão. São Paulo: OMS, 1995.

7. Santos JP, Mattos AP. Absenteísmo-doença na prefeitura municipal de Porto Alegre. Rev Bras Saúde Ocup. 2010;35(121):148-56.

8. Leão ALM, Barbosa-Branco A, Neto ER, Ribeiro CAN, Turchi MD. Absenteísmo-doença no serviço público municipal de Goiânia. Rev Bras Epidemiol. 2015;18(1):262-77.

9. Margrhraf MRS, Silva DV. Perfil do absenteísmo na prefeitura municipal de Curitiba de 01/01/2009 a 31/12/2009 [acesso em 2013 dez 20]. Disponível em: http://www.imap.curitiba. pr.gov.br/wp-content/uploads/2014/03/Perfil\%20do\%20 absente\%C3\%ADsmo\%20na\%2OPrefeitura\%20Municipal\%20 de\%20Curitiba\%20no\%2Oper\%C3\%ADodo\%20de\%20 01012009\%20\%C3\%A0\%2031122009.pdf

10. National Audit Office. Managing sickness absence in the States of Guernsey [acesso em 2013 dez 20]. Disponível em: <http://gov.gg/ CHttpHandler.ashx?id=5139\&p=0>.11. Bof de Andrade T, Souza MGC,
Simões MPC, Bof de Andrade F. Prevalência de absenteísmo entre trabalhadores do serviço público. Scientia Medica 2008;18(4):166-71.

12. Ribeiro CAN, Moreira D. Absenteísmo por lombalgia na prefeitura de Goiânia nos anos de 2008 e 2009. Fisioter Mov. 2014;27(3):349-59.

13. Silva DMPP, Marziale MHP. Absenteísmo de trabalhadores de enfermagem em um hospital universitário. Rev Latino-Am Enfermagem. 2000;8(5):44-51.

14. Silva LS, Pinheiro TMM, Sakurai E. Perfil do absenteísmo em um banco estatal em Minas Gerais: análise no período de 1998 a 2003. Ciênc Saúde Coletiva. 2008;13(Suppl 2):2049-58.

15. Reis JR, La Rocca PF, Silveira AM, Bonilla IML, Navarro i Giné A, Martín M. Fatores relacionados ao absenteísmo por doença em profissionais de enfermagem. Rev Saúde Pública. 2003;37(5):616-23.

16. Carneiro KLA. O impacto do absenteísmo em uma empresa de construção civil [acesso em 2013 dez20]. Disponível em: <repositorio. uniceub.br/bitstream/123456789/1021/2/21032561.pdf>

17. Saraiva ALB, Correa WSC, Nascimento FH, Azevedo TS. Qualidade do ar no município de Vitória-ES entre 2000-2009. Rev Geonorte. 2012:5:573-85

18. Castro HA, Hacon S, Argento R, Junger WL, Mello CF, Castioglioni Júnior $\mathrm{N}$, et al. Doenças respiratórias e poluição atmosférica no município de Vitória, Espirito Santo, Brasil. Cad Saúde Pública. 2007;23(Suppl. 4):S630-42.

19. Freitas CU,Junger W,Leon AP, Grimaldi R,Silva MAFR, Gouveia N. Poluição do ar em cidades brasileiras: selecionando indicadores de impacto na saúde para fins de vigilância. Epidemiol Serv Saúde. 2013;22(3):445-54.

Endereço para correspondência: Fábio Petersen Saraiva - Departamento de Medicina Especializada da Universidade Federal do Espírito Santo -

Avenida Marechal Campos, 1468 - Maruipe - CEP: 29047-105 - Vitória (ES)

Brasil - E-mail: fabiopetersen@yahoo.com.br 